\title{
Erratum to: Improved top-down control reduces oculomotor capture: The case of action video game players
}

\author{
Joseph D. Chisholm • Alan Kingstone
}

Published online: 14 April 2012

(C) Psychonomic Society, Inc. 2012

\section{Erratum to: Atten Percept Psychophys \\ DOI 10.3758/s13414-011-0253-0}

The abstract that appears below was inadvertently left out of the original article.

\begin{abstract}
Action video game players (AVGPs) have been demonstrated to outperform non-video-game players (NVGPs) on a range of cognitive tasks. Evidence to date suggests that AVGPs' enhanced performance in attentionbased tasks can be accounted for by improved top-down control over the allocation of visuospatial attention. Thus, we propose that AVGPs provide a population that can be used to investigate the role of top-down factors in key models of attention. Previous work using AVGPs has indicated that they experience less interfering effects from a
\end{abstract}

salient but task-irrelevant distractor in an attentional capture paradigm (Chisholm, Hickey, Theeuwes, \& Kingstone, 2010). Two fundamentally different bottom-up and topdown models of attention can account for this result. In the present study, we compared AVGP and NVGP performance in an oculomotor capture paradigm to address when and how top-down control modulates capture. In tracking eye movements, we acquired an explicit measurement of attention allocation and replicated the covert attention effect that AVGPs are quicker than NVGPs to attend to a target in the presence of a task-irrelevant distractor. Critically, our study reveals that this top-down gain is the result of fewer shifts of attention to the salient distractor, rather than faster disengagement after bottom-up capture has occurred. This supports the theory that top-down control can modulate the involuntary capture of attention.

The online version of the original article can be found at http://dx.doi.org/ 10.3758/s13414-011-0253-0.

J. D. Chisholm $(\bowtie) \cdot$ A. Kingstone

University of British Columbia,

Vancouver, British Columbia, Canada

e-mail: jchisholm@psych.ubc.ca 